\title{
Sportphysiotherapie für Hunde
}

\author{
Ganzheitliche tiermedizinische Untersuchung und \\ Behandllung von Sporthunden
}

Silke Meermann

\section{๑) Zusammenfassung}

Während die Sportphysiotherapie im Humanbereich und auch beim Pferd schon seit vielen Jahren eine wichtige Rolle bei der Betreuung von Sportpatienten spielt, wird diese in den letzten Jahren auch für Sporthunde immer mehr nachgefragt. Dabei setzt ein solches Arbeiten Kenntnisse über die entsprechenden körperlichen Anforderungen der verschiedenen Hundesportarten, sowie spezialisierte fachliche Kenntnisse und Fähigkeiten im Hinblick auf die Behandlungsmöglichkeiten voraus - die Arbeit mit Sporthunden ist für den Therapeuten jedoch in der Regel auch sehr motivierend, da in erster Linie mit gesunden und gut trainierten Patienten, sowie mit Besitzern gearbeitet wird, die in hohem Maße bemüht sind, die Therapievorschläge umzusetzen.

\section{Definitionen und Begriffsbestimmungen}

Unter dem Begriff der Physiotherapie werden physikalische bzw. physiologische äußerliche Anwendungen am Bewegungsapparat des Patienten zusammengefasst. Dabei umfasst die Physiotherapie verschiedene Teilbereiche: so kann je nach Zielsetzung der Behandlung zwischen kurativer physiotherapeutischer Behandlung, Rehabilitation und Prävention unterschieden werden [1]; es kann darüber hinaus eine Unterscheidung in orthopädisch-physiotherapeutische, neurologisch-physiotherapeutische und traumatologisch-physiotherapeutische Fälle erfolgen [4]. Im Hinblick auf die zur Anwendung kommenden Behandlungstechniken kann wiederum zwischen physikalischen Anwendungen im engeren Sinne (Physiotechnik), Manuellen Therapieformen und der Bewegungstherapie (Abb. 1) differenziert werden. Die physikalischen Therapieformen umfassen die Thermotherapie, die Hydrotherapie, die Elektrotherapie, die Photonenbzw. Lasertherapie, die Magnetfeldtherapie und den therapeutischen Ultraschall. Manuelle Therapieformen beinhalten die klassische Massage, die manuelle Lymphdrainage und die Manualtherapie (Gelenktraktion, -kompression und Translatori- sches Gleiten). In Deutschland werden zum Teil auch die Osteopathie und die Chiropraktik zu den manuellen Therapieformen gezählt, da auch hier ausschließlich die Hände als Behandlungsinstrumente eingesetzt werden. Allerdings sind sowohl die Osteopathie als auch die Chiropraktik Mitte des 19. Jahrhunderts in den USA als eigenständige Therapieformen entstanden und stark durch eine holistische Behandlungsphilosophie und ein bescheidenes Selbstverständnis des Therapeuten geprägt, sodass sie sich dadurch deutlich von anderen Therapieformen unterscheiden. Die Bewegungstherapie entspricht zum Teil der Krankengymnastik, wie sie im Humanbereich zur Anwendung kommt; allerdings sind beim Tier hier deutlich engere Grenzen gegeben, da eine abstrakte Übungsanleitung nicht möglich ist.

Im Humanbereich wird darüber hinaus klar differenziert, ob die entsprechenden Therapieformen von Physiotherapeuten mit geregelter Berufsausbildung bzw. Master-Studium, oder aber von Ärzten mit entsprechender Weiterbildung angewendet werden: bei der Anwendung
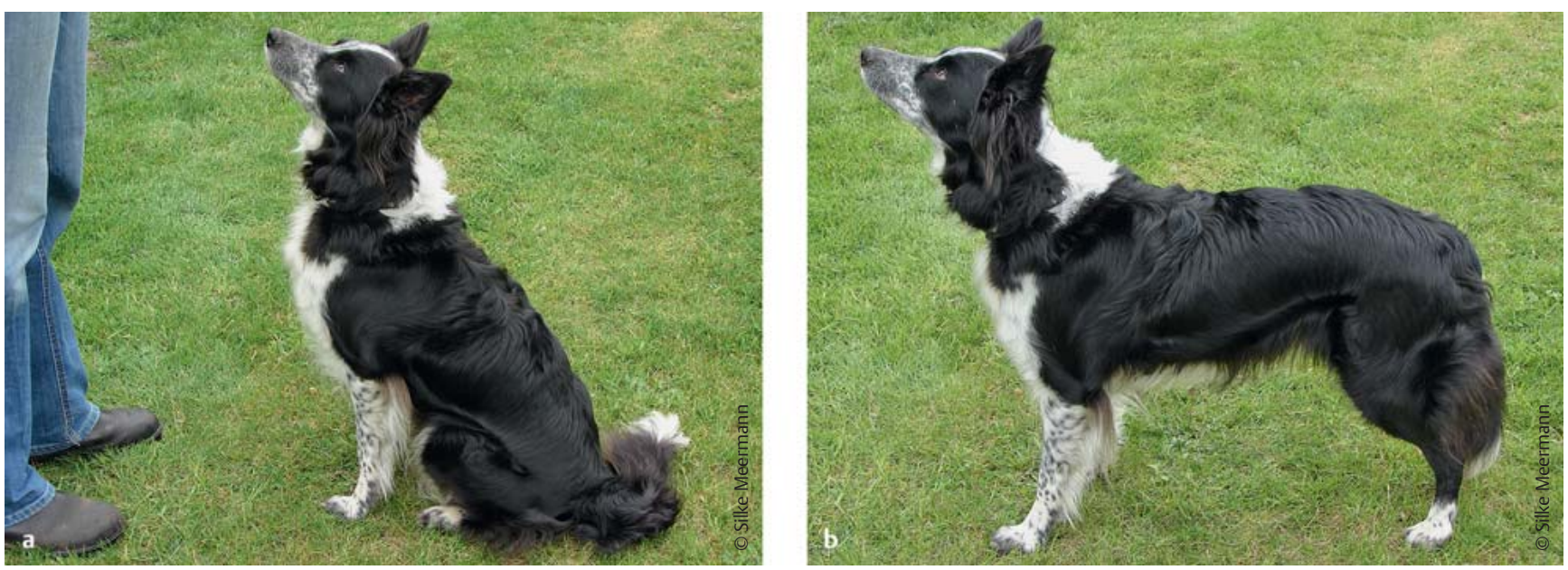

Abb. 1 a und b Sitz-Steh-Übergänge als eine Übung aus dem Bereich der aktiven Bewegungstherapie führen durch konzentrische und exzentrische Muskelarbeit zu einem Aufbau der Hinterhandmuskulatur. 

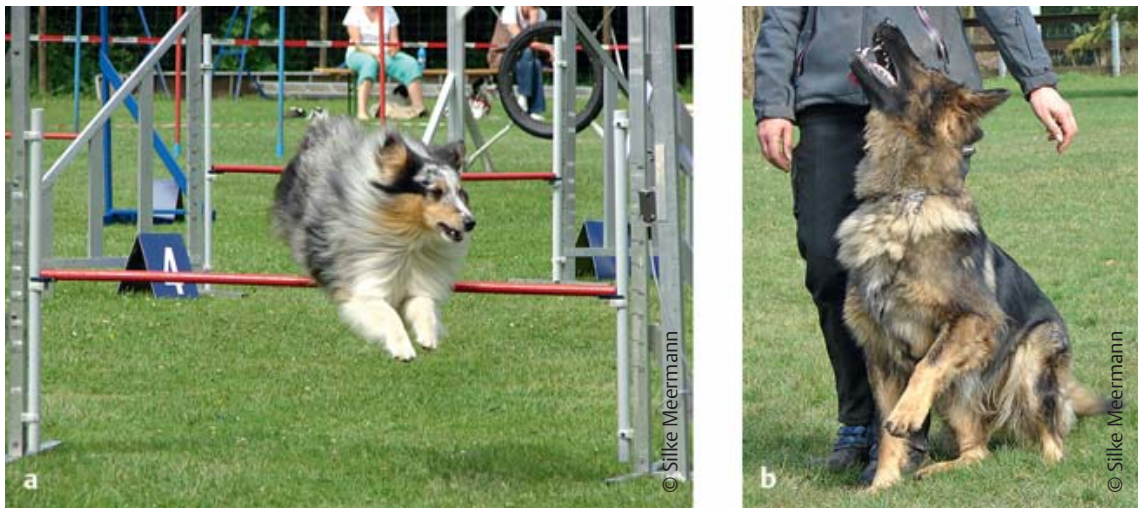

Abb. 2 a und b Die Sportphysiotherapie beschäftigt sich mit speziellen Fragestellungen, die sich aus der sportlichen Betätigung des Hundes ergeben. Im Agility stehen schnelle Sprungbelastungen im Vordergrund, bei der Fußarbeit ( $\mathbf{A b b}$. 2 b) wird die tonische Nackenreaktion ausgelöst.

durch Ärzte spricht man dann von physikalischer Medizin oder Sportmedizin. Diese Abgrenzung ist im Tierbereich durch die Zusatzbezeichnungen für Tierärzte auch möglich, wird jedoch häufig nicht so klar getroffen. Vielen Tierbesitzern ist nicht bewusst, dass sich hinter Begriffen wie „Tierphysiotherapeut" keine staatlich geregelte Berufsausbildung verbirgt. Der Begriff „Tierphysiotherapie“ ist für Besitzer jedoch verständlich und greifbar, während die „Zusatzbezeichnung Physikalische Medizin“ für viele Besitzer keine konkrete Bedeutung hat.

Die Sportphysiotherapie bzw. Sportmedizin beschäftigt sich mit der Untersuchung und Behandlung von Sportlern hier also Sporthunden, die in einer oder mehreren Hundesportarten geführt werden ( Abb.2). Sie umfasst dabei das gesamte Spektrum physikalischer und physiotherapeutischer Behandlungsmethoden. Übertragen aus dem Humanbereich zielt die Sportphysiotherapie darauf ab, die Gesundheit des Hundes zu fördern und zur Gesunderhaltung der Hundepopulation durch sportliche Aktivität beizutragen. Dies setzt auch ein entsprechendes Selbstverständnis des Therapeuten voraus. Im Einzelnen definiert die Sportphysiotherapie die folgenden vier Behandlungsziele:

1. Verletzungsprävention,

2. akute Intervention,

3. Rehabilitation,

4. Leistungssteigerung.

\section{Verletzungsprävention}

Um Verletzungen vorbeugen zu können, müssen zunächst Verletzungsrisiken erkannt werden; sind diese Risiken identi- fiziert, steht die Aufklärung von Hundesportler und Trainer im Vordergrund. Verletzungsprävention muss sportartspezifisch geschehen - das bedeutet, dass der Therapeut hier gute Kenntnisse über die körperlichen Anforderungen, aber auch über die jeweiligen Reglements der einzelnen Sportarten besitzen muss. Darüber hinaus sind natürlich individuelle Faktoren wie Alter, Geschlecht, aber auch Ernährungszustand und individuelle Besonderheiten in Bezug auf den Körperbau (z.B. besonders steile Winkelung der Gliedmaßengelenke etc.) zu berücksichtigen. Die Kommunikation mit Hundesportlern und Trainern spielt bei der Prophylaxe eine extrem wichtige Rolle, da die präventiven Maßnahmen in Training und Wettkampf umgesetzt werden müssen, ohne dass der Therapeut unmittelbar zugegen ist. Es genügt in der Regel nicht, den einzelnen Hundesportler von einer Trainingsmaßnahme (z.B. zielgerichtetes Warm-up vor Training und Wettkampf) zu überzeugen, da er diese meist nur umsetzen kann, wenn der Trainer hierfür Zeit und Raum zur Verfügung stellt. Workshops für Gruppen oder Vereine mit Sportlern und Trainern zusammen stellen daher eine gute Möglichkeit dar, um solche Informationen zur Verletzungsprävention zu multiplizieren!

\section{Akute Intervention}

Im Bereich der akuten Interventionsmöglichkeiten unterscheidet sich die Sportphysiotherapie nicht von der allgemeinen Physiotherapie. Hier geht es darum, bei akuten Verletzungen oder nach chirurgischen Eingriffen möglichst frühzeitig die physiologischen Prozesse, die in den frühen Wundheilungsphasen ablaufen, zu unterstützen. Hier stehen Techniken wie die manuelle Lymphdrainage und das PECH-Prinzip ( $\mathrm{P}=$ Pause, Sportpause; $\mathrm{E}=$ Eis; Kryotherapie; $\mathrm{C}=$ Compression; Kompression; $\mathrm{H}=$ Hochlagern; beim Hund von der praktischen Umsetzung her oft schwierig) im Vordergrund.

\section{Rehabilitation}

Hinter dem Begriff der Rehabilitation verbirgt sich die gezielte Rückführung möglichst auf das zuvor vorhandene Leistungsniveau nach einer Verletzung oder Operation. Hier muss immer zunächst kritisch hinterfragt werden, ob dieses Ziel tatsächlich erreichbar bzw. im Hinblick auf die Gesundheit des Hundes erstrebenswert ist. So ist es beispielsweise nach einer Ruptur des kranialen Kreuzbandes im Kniegelenk trotz chirurgischer Versorgung und physiotherapeutischer Behandlung in der Regel nicht möglich, einen Hund, der zuvor eine schnelle Sprungsportart auf Leistungssportniveau (z.B. Agility; Leistungsklasse A3) ausgeübt hat, wieder an diese Belastung heranzuführen. Hier kann es für die langfristige Gesunderhaltung des Hundes notwendig sein, mit dem Besitzer alternative sportliche Beschäftigungsmöglichkeiten zu erörtern, die eine weniger starke Gelenkbelastung bedingen (z.B. Obedience, Mantrailing etc.). Im Hinblick auf eine erfolgreiche Rehabilitation muss für jeden Patienten ein individuelles Therapiekonzept erarbeitet werden. So können bei verschiedenen Hunden mit derselben schulmedizinischen Diagnose wie z.B. „mittels TPLO versorgte Ruptur des kranialen Kreuzbandes des rechten Kniegelenkes" unterschiedliche Probleme im Vordergrund stehen: während bei einem Patienten die Kniegelenks-Extension durch eine Kapselfibrose infolge unzureichender Mobilisation eingeschränkt ist, besteht bei einem anderen Patienten eine Einschränkung der Kniegelenks-Flexion aufgrund einer noch vorhandenen Gelenkschwellung. Bei einem dritten Patienten kann dagegen eine ipsilaterale Lumbalgie mit muskulärem Hartspann der paravertebralen Muskulatur der Lendenwirbelsäule zu Beschwerden führen, während bei einem vierten Patienten mit einer fehlenden Belastung der operierten Gliedmaße die Gangschulung im Hinblick auf das phy- 
siologische Bewegungsmuster behandelt werden muss.

\section{Leistungssteigerung}

Ein weiteres Behandlungsziel der Sportphysiotherapie ist die Leistungssteigerung im Sport; dabei ist diese per definitionem bereits eigentlich Ziel eines jeden sportlichen Trainings und fällt somit in erster Linie in den Aufgabenbereich des Hundesport-Trainers. Problematisch in Bezug auf die Leistungssteigerung im Hundesport ist oftmals die Objektivierung anhand messbarer Trainings- und Wettkampfziele. Dies ist bei Rennsportarten über die Messung der Geschwindigkeit zwar relativ einfach möglich, bei vielen anderen Sportarten (z.B. Agility, Obedience,

\section{Ganzheitlichkeit}

Der Begriff der Ganzheitlichkeit umfasst verschiedene Aspekte. So bedeutet er zum einen, dass ein Individuum als eine Einheit aus Körper, Seele und Geist begriffen wird. Bei der ganzheitlichen Behandlung von Sporthunden findet dies beispielsweise Berücksichtigung, wenn ein Hund sich in einem Agility-Wettkampf an der A-Wand verletzt hat: die Behandlung umfasst die Therapie der Verletzung auf körperlicher Ebene, sollte aber auch Trainingsansätze auf der Verhaltensebene beinhalten, um zu verhindern, dass der Hund ein erlerntes Vermeiden in Bezug auf das entsprechende Gerät entwickelt. Das Prinzip der Ganzheitlichkeit spiegelt sich darüber hinaus in den Holistischen Therapieformen ganzheitlicher Behandlungsansatz, dass verschiedene Therapieformen in den Behandlungsplan integriert werden: so spielen bei der sportphysiotherapeutischen Behandlung eines Patienten neben den klassisch-medizinischen Gebieten der Orthopädie, Neurologie und inneren Medizin natürlich auch die Physiotherapie, sowie die Physikalische Medizin eine wichtige Rolle; in vielen Fällen kommen aber auch Techniken zur Anwendung, die der Osteopathie oder Chiropraktik entstammen; diese können je nach Problemstellung auch mit der Akupunktur, der Phytotherapie oder der Biologischen Tiermedizin kombiniert werden. Viele Hundesportler suchen je nach Fragestellung jedoch auch verschiedene Therapeuten auf, sodass hier
Turnierhundsport) werden den Hunden jedoch viele verschiedene, z.T. sehr komplexe Aufgaben abverlangt und oftmals spielt die Zusammenarbeit mit dem menschlichen Teampartner eine entscheidende Rolle, sodass eine Steigerung der sportlichen bzw. körperlichen Leistung des Hundes oft nur schwer zu erfassen ist. Bei diesen komplexen Sportarten ist es in vielen Fällen auch nicht die körperliche Leistungsfähigkeit des Hundes, die den limitierenden Faktor im Hinblick auf den Wettkampferfolg darstellt, sodass eine Steigerung der körperlichen Leistungsfähigkeit hier nicht unbedingt zu besseren Wettkampfleistungen führt (\$ Abb. 3). wieder: dies sind Therapieformen, die sich an einem holistischen, anatomischen System orientieren. Beispielhaft kann hier die Faszienbehandlung genannt werden: die Faszien durchziehen den gesamten Körper und verbinden dadurch alle Strukturen des Bewegungsapparates, aber auch alle inneren Organe miteinander. So können fasziale Fehlspannungen auf der einen Seite Ursache dafür sein, dass Bewegungseinschränkungen nicht nur auf eine Gliedmaße beschränkt bleiben, sondern sekundäre Beschwerden in weit entfernten Körperregionen hervorrufen; auf der anderen Seite wird so die Behandlung mittels Faszientechniken eingesetzt, um nicht nur das primär verletzte Gelenk oder Körperteil, sondern den gesamten Körper mit zu behandeln. Nicht zuletzt bedeutet ein die Kommunikation mit dem Besitzer und unter Umständen auch mit den anderen Therapeuten zur möglichst optimalen Abstimmung der Therapieformen aufeinander unerlässlich ist.

\section{Die Fünf Haupt- beanspruchungsformen}

Die fünf Hauptbeanspruchungsformen bilden die Grundlage der sportlichen Trainingslehre. Eine Beanspruchung ist die individuelle Reaktion des Körpers auf eine Belastung; sie ist messbar an körperlichen Reaktionen wie beispielsweise der Herzoder Atemfrequenz. Auf eine Beanspruchung reagiert der Körper zunächst mit einer Ermüdung; anschließend erfolgt die Optimierung des körperlichen Zustandes 


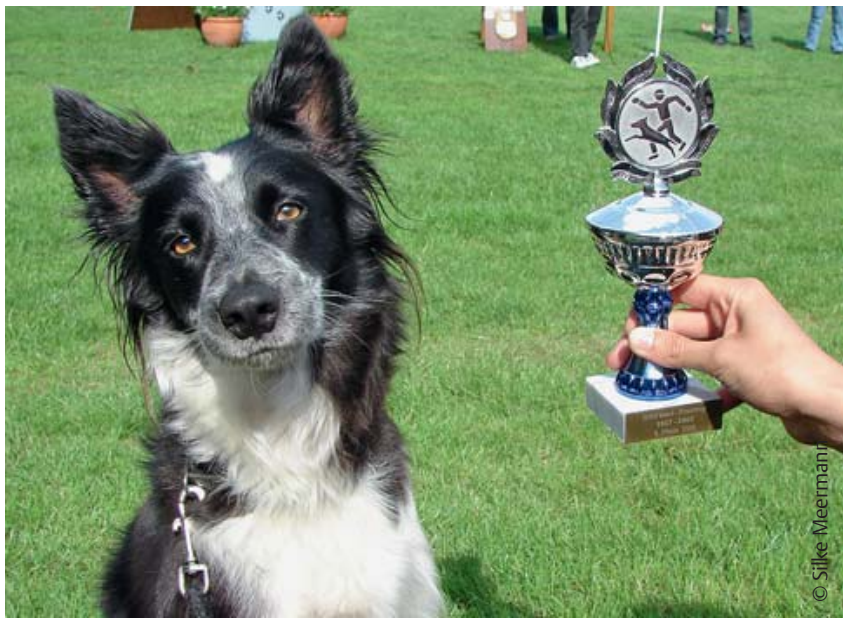

Abb. 3 Die Steigerung der körperlichen Leistungsfähigkeit des Hundes führt nicht immer auch zu einem besseren Abschneiden im Wettkampf.

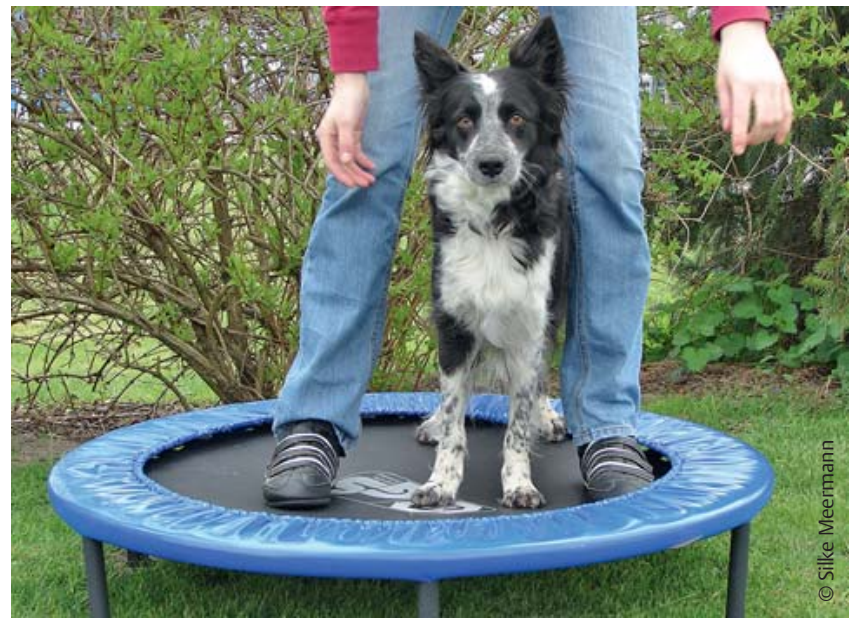

Abb. 4 Stehen auf einer labilen Unterlage, wie hier einem Zimmertrampolin, auf dem sich der Besitzer bewegt, trainiert die Fähigkeit der Koordination. durch Anpassungsprozesse. Die fünf Hauptbeanspruchungsformen werden durch die Eigenschaften Ausdauer, Kraft, Schnelligkeit, Koordination und Beweglichkeit repräsentiert; sie können durch ein entsprechend auf diese Fähigkeit ausgerichtetes Training verbessert werden.

1. Ausdauer stellt die psychisch-physische Fähigkeit dar, Widerstand gegen Ermüdung zu leisten. Bestimmend für die Ausdauer sind die Herz-KreislaufFunktion, der Muskelstoffwechsel, aber auch die neuro-muskuläre Koordination. Eine gute Ausdauer wird beispielsweise bei Schlittenhunderennen, bei Geländeläufen über größere Distanzen, aber auch im Mantrailing und bei der Rettungshundearbeit benötigt.

2. Kraft bezeichnet die Fähigkeit des Nerv-Muskelsystems, durch Innervations- und Stoffwechselprozesse mit Hilfe von Muskelkontraktionen Widerstände zu überwinden; dabei wird biochemisch gespeicherte Energie in mechanische Energie umgewandelt. Die Definition des Kraftbegriffes in der Trainingslehre geht somit über die rein physikalische Kraftdefinition (Kraft = Masse $\times$ Beschleunigung) hinaus. Man unterscheidet verschiedene Kraftformen wie die Maximalkraft, die Schnellkraft und die Kraftausdauer. Vor allem Schnellkraft wird in Sprungsportarten wie im Agility, aber auch bei der Schutzhundarbeit und bei Sprintrennen benötigt.

3. Schnelligkeit ist die Fähigkeit, auf einen Reiz hin möglichst schnell zu reagieren und/oder Bewegungen bei geringem
Widerstand mit höchster Geschwindigkeit auszuführen. Auch diese Eigenschaft wird bei allen schnellen Sportarten benötigt. Diese Eigenschaft ist im Wesentlichen genetisch bedingt und lässt sich durch Training nur innerhalb relativ enger Grenzen steigern.

4. Die Koordination beschreibt das Zusammenwirken von ZNS und Muskulatur innerhalb gezielter Bewegungsabläufe; man unterscheidet die intra- und intermuskuläre Koordination. Diese Fähigkeit geht vor allem auf Lernerfahrungen im Junghundalter zurück. Dementsprechend sollten Hunde, die später in koordinativ anspruchsvollen Sportarten (Agility, aber auch Trümmersuche) geführt werden, früh ein entsprechendes Propriozeptions- und Koordinationstraining durchlaufen (verschiedene Untergründe, Schaukeln, Wackelbretter, Balance-Bretter etc.) (ه Abb. 4).

5. Als Beweglichkeit bezeichnet man die Fähigkeit, willkürliche Bewegungen mit der erforderlichen Schwingungsweite ausführen zu können. Die Gesamtbeweglichkeit eines Individuums wird dabei von der Beweglichkeit der einzelnen Gelenke bestimmt. Zur Erhaltung einer guten Beweglichkeit sollten vor einer sportlichen Belastung aktive Bewegungsübungen abgerufen werden; im Anschluss an die Belastung können passive Dehnungsübungen erfolgen; diese sollten immer an die jeweilige Sportart, aber auch an eventuell bestehende individuelle Bewegungseinschränkungen angepasst sein.

\section{Trainingsplanung}

Ein relevanter Trainingsreiz muss so gewählt werden, dass er etwas über die Alltagsbelastung hinausgeht, damit der Körper mit den gewünschten Anpassungsprozessen reagiert. Wird beispielsweise mit einem Ausdauertraining begonnen, muss die Laufstrecke länger sein oder schneller absolviert werden, als dies bei den normalen Spaziergängen der Fall ist. Auf einen solchen Trainingsreiz reagiert der Körper zunächst mit Ermüdung, dann schließen sich die entsprechenden Adaptationsvorgänge an. Dabei werden die Körperfunktionen für einen kurzen Zeitraum optimiert - dies bezeichnet man als Phase der Superkompensation. Um durch ein Training insgesamt eine Leistungssteigerung zu erreichen, muss der nächste Trainingsreiz in dieser Phase der Superkompensation erfolgen. Für die Mehrzahl der Gewebe ist dies zwei bis drei Tage nach dem vorangegangenen Reiz der Fall. Neben dieser Trainingssteuerung mit Hilfe kurzer zeitlicher Zyklen sollte darüber hinaus auch eine Jahresperiodisierung stattfinden, sodass das Training auf die Saisonhöhepunkte im Sportjahr in eine Vorbereitungsperiode, eine Wettkampfperiode und eine Übergangsperiode unterteilt werden kann. Um ein Übertrainingssyndrom zu vermeiden, sollte jedem Hund eine mindestens einmonatige zusammenhängende Wettkampfpause pro Jahr gegönnt werden! 


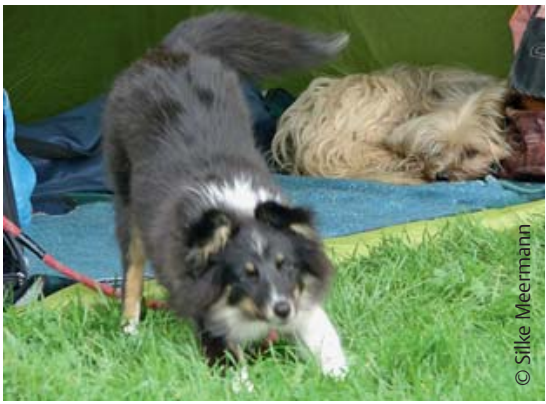

Abb. 5 Aktive Bewegungsübungen sind ein wichtiger Teil des Aufwärmprogrammes im Training und im Wettkampf.

\section{Bedeutung des \\ Auf- und Abwärmens}

Dem Aufwärmen vor und dem Abwärmen nach einer Belastung kommt eine besondere Bedeutung im Hinblick auf die Vermeidung akuter Sportverletzungen einerseits, aber auch chronischer Verschleißerscheinungen wie Arthrosen und Spondylosen andererseits zu.

Die positiven Effekte des Aufwärmens lassen sich auf verschiedene physikalische und physiologische Zusammenhänge zurückführen: so kommt es allgemein zu einer Beschleunigung aller Stoffwechselprozesse im Körper (Reaktions-Geschwindigkeits-Temperatur-Regel; mit einer Erhöhung der Körpertemperatur um $1^{\circ} \mathrm{C}$ laufen alle enzymatischen Prozesse etwa 13\% schneller ab). Die höhere Temperatur bedingt ebenfalls verbesserte elastische Eigenschaften von Muskulatur und passiven Bindegewebsstrukturen, sodass das Risiko für Muskelfaser-, Sehnen- und Bänderrisse sinkt. Im Bereich der Muskulatur sorgt die verbesserte Durchblutungssituation vor allem für eine bessere Sauerstoffversorgung; die erwärmte Arbeitsmuskulatur kann darüber hinaus schneller kontrahieren und auch schneller relaxieren. $\mathrm{Zu}$ den positiven Effekten auf das HerzKreislaufsystem zählen eine geringere Blutdrucksteigerung mit einem geringeren Anstieg der Vor- und Nachlast am Herzen sowie ein geringeres Risiko für EKG-Abweichungen und Herzrhythmus-Störungen; dies ist vor allem auch auf die verbesserte Sauerstoffversorgung des Herzmuskels zurückzuführen. Auch das ZNS wird durch ein gezieltes Aufwärmen aktiviert und es kommt zu einer geringeren Startverzögerung, was vor allem bei Sprintsportarten von Vorteil ist. Darüber hinaus bietet das Aufwärmen zudem die Möglich-

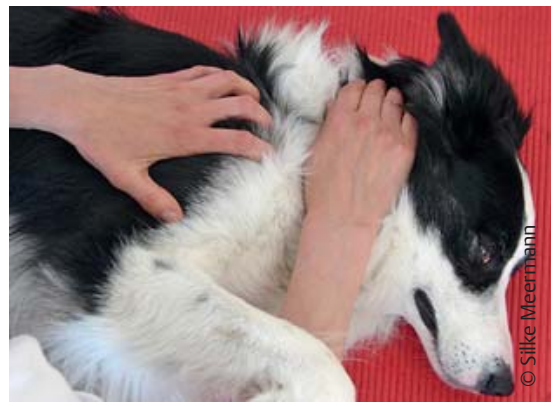

Abb. 6 Die Lockerungsmassage als Abschluss des Abwärmens genießen die meisten Hunde sehr.

keit, mit einer fremden Umgebung beim Wettkampf „warm zu werden“ und der Hundesportler kann bereits beim Aufwärmen gezielt versuchen, die Motivationslage des Hundes beruhigend oder aktivierend zu beeinflussen. In der Summe verbessert ein Aufwärmen vor einer Belastung die Leistungsfähigkeit des Hundes vor allem durch eine geringere Startverzögerung, eine schnellere Muskelarbeit, eine verbesserte Muskeldurchblutung, sowie eine verbesserte Konzentration und Motivation.

Der Ablauf des Aufwärmens gestaltet sich dabei prinzipiell immer ähnlich mit einem Allgemeinen Aufwärmen, an das sich ein Spezielles Aufwärmen mit sportartspezifischen Bewegungsübungen anschließt ( Abb.5). Das Allgemeine Aufwärmen besteht aus einem lockeren Traben über mindestens zehn Minuten; dabei kann der Hund am Fahrrad geführt werden oder aber der Besitzer läuft gemeinsam mit dem Hund. Daran schließen sich aktive Stretchings und muskelaktivierende Übungen an. Der Vorteil dieser aktiven Bewegungsübungen besteht in einer Verbesserung der Beweglichkeit ohne dass es zu einer vor der Belastung unerwünschten Senkung des Muskeltonus kommt. Außerdem ist bei aktiven Übungen die Gefahr des Überdehnens wesentlich geringer als bei passiven Übungen. Aktive Übungen können darüber hinaus die Konzentration des Hundes fördern und seine Motivationslage positiv beeinflussen. Das Spezielle Aufwärmen umfasst Übungen, die den Hund auf die spezifischen Bewegungsabläufe der jeweiligen Sportart vorbereiten; das Spezielle Aufwärmen im Agility sollte also beispielsweise Sprünge, Richtungswechsel und Slalombewegungen beinhalten. Das Aufwärmen sollte insgesamt etwa 15-20 Minuten dauern; da- bei sollte eine Körperkerntemperatur von knapp $40^{\circ} \mathrm{C}$ und ein Arbeitspuls von etwa 150 Schlägen pro Minute erreicht werden.

Ohne ein gezieltes Abkühlen bleibt nach einer Belastung eine große Blutmenge im Bereich der Arbeitsmuskulatur zurück; dadurch kann es zu Kreislaufproblemen kommen und der Abtransport von Stoffwechselendprodukten verzögert sich. Die wichtigsten positiven Effekte des Abkühlens sind somit eine bessere Entschlackung der Muskulatur, eine Senkung des Muskeltonus und ein schnellerer Abtransport und Abbau der körpereigenen Stresshormone Adrenalin und Cortisol.

Auch der Ablauf des Abwärmens ist prinzipiell immer gleich: zunächst erfolgt ein lockeres Auslaufen erst im Trab und dann im Schritt für insgesamt ebenfalls 10-15 Minuten; das Abwärmen ist abgeschlossen, wenn die Überprüfung der Kreislauffunktionen annähernd wieder Ruhewerte ergibt. Dann schließen sich passive Dehnübungen und detonisierende Massagetechniken ( Abb. 6) an.

\section{Basis-Sprungtraining}

Am Beginn der Ausbildung jedes Hundes in einer Sprungsportart (z. B. Agility, Schutzhundausbildung, Turnierhundsport etc.) sollte ein gezielter, biomechanischer Aufbau der Sprungtechnik stehen. Ohne ein solches Training überwinden die meisten Hunde Sprünge oft mit einer relativ gestreckten Hals- und Brustwirbelsäule, dadurch kommt es über die tonische Nackenreaktion [5] zu einer starken Flexion der großen Gelenke der Hintergliedmaßen. Eine solche Technik ist vor allem dann, wenn schnelles Überwinden vieler Hindernisse auf gebogenen Linien, wie es beispielsweise im Agility gefragt ist, oft nachteilig. Aus diesem Grund sollten die Hunde zusätzlich eine bogenförmige Sprungtechnik mit flektierter Wirbelsäule erlernen ( $\$$ Abb. 7), mit der auch höhere Hindernisse gut überwunden werden können [6]. Die biomechanischen Lernziele eines solchen Sprungtrainings sind das Erlernen einer stabilen Landung, die Vermeidung unnötiger Drehmomente und die Umlenkung der Landungsenergie in die erneute Vorwärtsbewegung. Ein solches Sprungtraining wird in mehrere Einzelschritte zerlegt: 


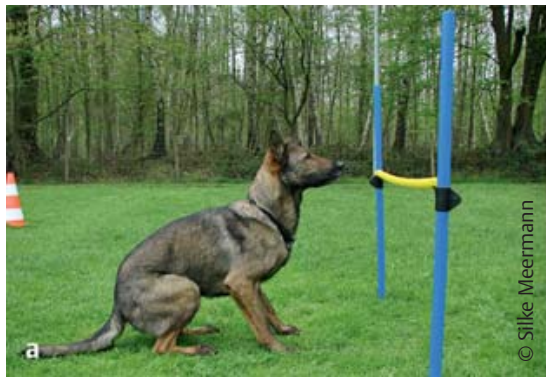

Abb. 7a bis c Training einer runden Sprungtechnik.

n Schritt 1: der Hundesportler hockt seitlich von der Hürde, der Hund springt über die noch sehr niedrig liegende Stange hin und her und wird mit Leckerchen am Boden bei der Landung bestätigt

- Schritt 2: die Hürde wird schrittweise höher gelegt; der Hund wird weiter vom hockenden Hundesportler am Boden belohnt

- Schritt 3: der Hundesportler hockt jetzt nicht mehr, sondern steht neben der Hürde und wirft das Leckerchen auf den Boden auf der Seite, wo der Hund landet

n Schritt 4: der Hund wird jetzt nicht mehr direkt vor der Hürde positioniert, sondern muss sich seinen Abstand und Absprungpunkt nun selbst suchen; das Leckerchen wird an verschiedene, weiter entfernte Punkte hinter der Hürde geworfen

- Schritt 5: nun variiert der Besitzer seine eigene Position in Bezug auf den Hund und in Bezug zur Hürde
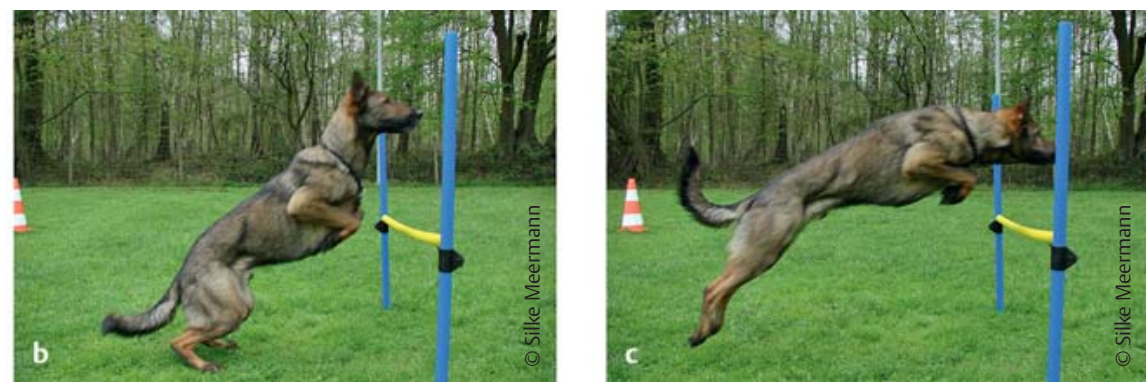

\section{Fallbeispiel: „Foppe ${ }^{6}$ Leistungsabfall im Agility}

Signalement und Vorbericht: „Foppe“ ist ein 5-jähriger, nicht kastrierter SheltieRüde, der im Agility in der höchsten Leistungsklasse (A3) geführt wird. Seit mehreren Wochen weigert sich der Hund nun, über die eigentlich geforderte Hürdenhöhe zu springen. In den Tagen nach einer Trainings- oder Wettkampfbelastung fällt der Besitzerin einer erhöhte Berührungsempfindlichkeit im Bereich der Lendenwirbelsäule auf; bei Druck am lumbosakralen Übergang setzt sich der Hund sofort ab. Eine aktuelle Röntgenuntersuchung der Wirbelsäule beim Haustierarzt ergab keine besonderen Befunde, die Hüften des Hundes waren im Alter von 12 Monaten geröntgt worden und hatten eine Einstufung als „HD-frei“ ergeben. Der Rüde hatte nun über 14 Tage ein nicht-steroidales Antiphlogistikum in Standard-Dosierung erhalten; während dieser Zeit schien er beschwerdefrei; nach Absetzen des Medikaments traten jedoch die gleichen Probleme wieder auf wie zuvor.

\section{Sportphysiotherapeutische Erstbefundung}

In der Klinischen Allgemeinuntersuchung sowie in der Neurologischen Untersuchung wurden keine abweichenden Befunde erhoben. In der Gangbildbetrachtung fiel vor allem im Trabtempo ein mit der Hinterhand nach rechts versetztes Gangbild („Crabwalking“) auf; im SchrittTempo zeigte der Hund über weite Strecken Passgang; dies war der Besitzerin zuvor nicht aufgefallen. Die Druck- und Triggerpunkt-Untersuchung ergab eine geringgradige Schmerzhaftigkeit an beiden Hüftgelenkspunkten (MA 31); der lumbosakrale Übergang war nicht druckempfindlich. Die Gelenkfunktionsprüfung ergab beidseits eine muskulär eingeschränkte Hüftextension bei einer ebenfalls beidseits bestehenden Empfindlichkeit des M. iliopsoas. Es bestand eine Gelenkdysfunktion im Bereich des rechten Iliosakralgelenkes im Sinne einer Kaudo-Dorsal-Rotation sowie eine Gelenkdysfunktion im Segment L5-L6.
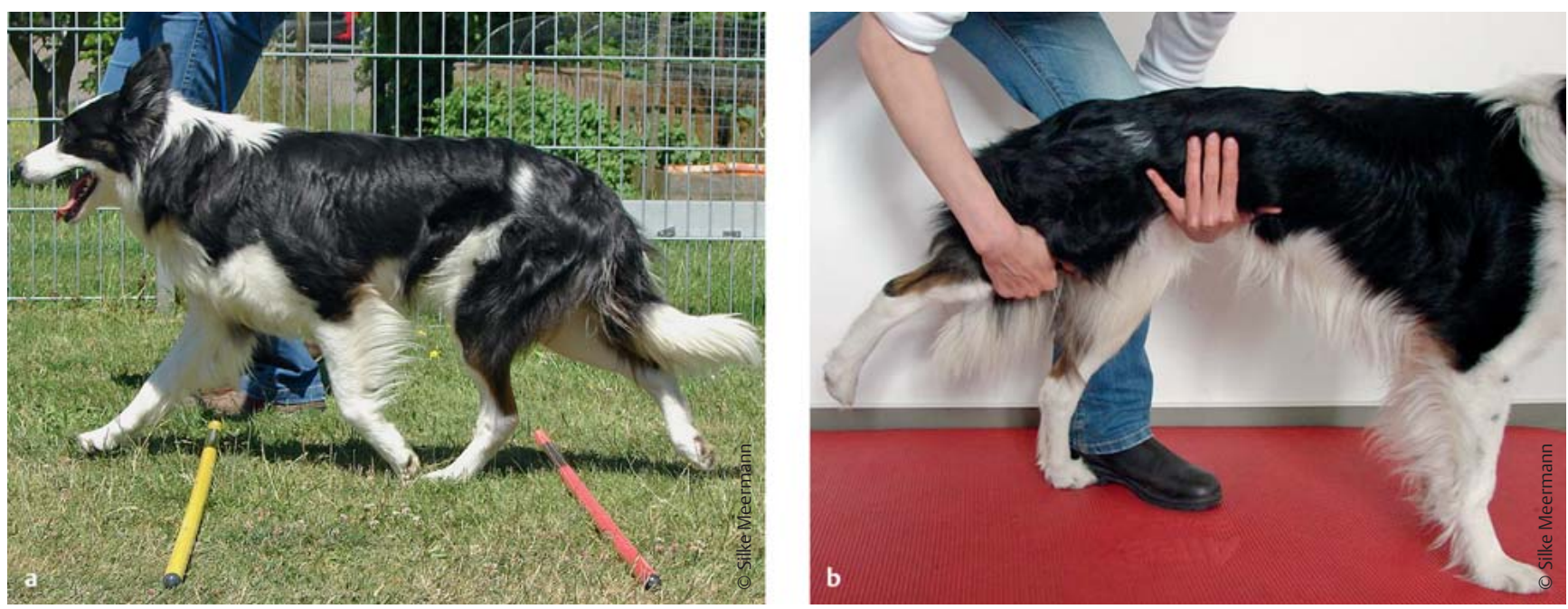

Abb. 8 a und b Die Besitzerin von „Foppe“ wurde angeleitet, ein regelmäßiges Trabtraining zu absolvieren, sowie täglich passiv die Hüftbeugemuskulatur zu dehnen. 


\section{Sportphysiotherapeutische Behandlung und Therapieplanung}

In der ersten Sitzung wurden die Gelenkdysfunktionen mittels chiropraktischer Impulstechniken behandelt; im Anschluss erfolgte eine Behandlung des myofaszialen Systems mit osteopathischen, indirekt-direkten Techniken. Die Hüftbeuger wurden mit detonisierenden Massagetechniken behandelt. Bei der anschließenden Kontrolle zeigten sich die Hüftgelenkspunkte nicht mehr empfindlich und auch der Umfang der Hüftgelenksextension hatte sich deutlich verbessert.

Die Besitzerin wurde angehalten, den Hund bis zur darauffolgenden Therapiesitzung eine Woche später nicht springen zu lassen; sie wurde angeleitet, mit dem Hund ein Trabtraining zu beginnen, sowie mindestens einmal täglich die Hüftbeuger passiv zu dehnen (\$Abb.8). Es wurde kritisch über eine Überweisung in eine Tierklinik zur weiteren Bildgebung zum Ausschluss eines röntgenologisch nicht darstellbaren Lumbosakralen StenoseSyndroms beraten; dies lehnte die Besitzerin jedoch aus Kostengründen ab.

\section{Verlauf}

„Foppe“ wurde über insgesamt zwei Monate lang zunächst wöchentlich, dann alle zwei Wochen in der Praxis behandelt; dabei stand das Lösen von Gelenkdysfunktionen, sowie die Behandlung mit myofaszialen Techniken im Vordergrund. Nach zwei Wochen begann die Besitzerin mit Foppe dreimal wöchentlich ein leichtes Sprungtraining durchzuführen, dabei wurden die Hindernisse zunächst nur auf 50\% der eigentlichen Höhe gelegt; Wendungen und andere Hindernisse wurden zunächst komplett weggelassen. Über insgesamt vier Wochen wurde dann die Sprunghöhe schrittweise wieder auf die eigentliche Wettkampfhöhe gesteigert und die übrigen Hindernisse wurden nach und nach wieder in das Training mit eingebaut. Vor jedem Training absolvierte die Besitzerin mit Foppe ein Aufwärmtraining und nach jeder Belastung ein Abwärmen inklusive passiver Dehnübungen insbesondere für die Muskulatur der Hintergliedmaßen. Nach knapp drei Monaten konnte der Rüde wieder am normalen Training und nach knapp vier Monaten wieder an Wettkämpfen teilnehmen.

Überlegungen zum Patho-Mechanismus: Bei „Foppe“ hatte die schmerzhafte, muskuläre Einschränkung der Hüftextension wahrscheinlich dazu geführt, dass der Hund im Sprung diese fehlende Extensionsbewegung über eine stärkere Streckung des lumbosakralen Übergangs bzw. der kaudalen Lendenwirbelsäule kompensiert hatte. Diese Überlastung führte in der Folge zu Dysfunktionen im Bereich der Iliosakralgelenke und der Lendenwirbelsäule mit einer erhöhten Berührungsempfindlichkeit in diesem Bereich. Da sich dieser Prozess bereits über Wochen verfestigt hatte, nahm auch die Therapie sowie die Rückkehr zum ursprünglichen Leistungsniveau mehrere Wochen in Anspruch.

\section{Fazit}

Übergeordnetes Behandlungsziel der Sportphysiotherapie ist die Gesunderhaltung durch Förderung sportlicher Aktivität. Das Therapiespektrum umfasst alle Techniken der Physiotherapie; darüber hinaus kommen weitere ganzheitliche Behandlungsformen zum Einsatz. Die erfolgreiche Behandlung von Sporthunden setzt Kenntnisse verschiedener Hundesportarten und deren spezifischer Belastungen voraus; außerdem muss eine gezielte Trainingsplanung unter Berücksichtigung physiologischer Aspekte erfolgen. Eine gute Zusammenarbeit von Hundeführer, Trainer und Therapeut ist dabei unerlässlich.

\section{() Summary}

\section{Canine sports physiotherapy: Holistic Veterinary examination and treatment of sporting dogs}

The underlying aim of canine sports physiotherapy is maintaining health by promoting sportive activities. Therapeutic interventions cover all kinds of physiotherapeutic as well as holistic therapies. For a successful treatment of the canine athlete a good knowledge of the most common dog sports as well as their main impacts on the canine body is essential. When retraining a dog, physiological aspects have to be considered. A good cooperation between dog handler, dog trainer and therapist is also necessary.

\section{$\checkmark$ Key words}

canine sports physiotherapy - therapeutic interventions - holistic therapy

\section{(- Literatur}

[1] Alexander CS, Hrsg. Physikalische Therapie für Kleintiere. Stuttgart: Parey; 2004

[2] Einsingbach T, Klümper A, Biedermann L. Sportphysiotherapie und Rehabilitation. Stuttgart: Georg Thieme; 1992

[3] Eitner D, Kuprian W, Meissner L, Ork H. SportPhysiotherapie. Stuttgart - New York: Gustav Fischer; 1990

[4] Hohmann M, Hrsg. Physiotherapie in der Kleintierpraxis - von der Befundung zum Therapieplan. Stuttgart: Sonntag; 2008

[5] Jaggy A, Hrsg. Atlas und Lehrbuch der Kleintierneurologie. 2. Aufl. Hannover: Schlütersche; 2007

[6] Mecklenburg L. Developing Jumping Skills. Clean Run Productions LLC South Hadley; 2007

[7] Zink M, van Dyke C und JB, Hrsg. Canine Sports Medicine and Rehabilitation. Ames lowa: Wiley-Blackwell; 2013

\section{Online zu finden unter}

http://dx.doi.org/10.1055/s-0035-1568189

\section{() Dr. med. vet. Silke Meermann}

Zusatzbezeichnung Physiotherapie und Rehabilitation bei Kleintieren

EAVC- und IVCA-zertifizierte

Veterinär-Chiropraktorin

Tierarztpraxis Am Schlagbaum

Am Schlagbaum 2a

59192 Bergkamen

E-Mail: info@tierarztpraxis-am-schlagbaum.de

1997-2002 Studium der Tiermedizin an der TiHo Hannover; 2002 Approbation als Tierärztin; 2005 Abschluss der Weiterbildung Veterinärchiropraktik an der European Academy for Veterinary Chiropractic (EAVC); Prüfung und Zertifizierung durch die International Veterinary Chiropractic Association (IVCA); 2008 Gründung der Tierarztpraxis Am Schlagbaum in Bergkamen gemeinsam mit Britta Westermann; ebenfalls 2008 Abschluss der Weiterbildung Physiotherapie und Physikalische Medizin für Kleintiere am Vierbeiner RehaZentrum, Bad Wildungen und Abschluss der Zusatzausbildung Canine Osteopathie am FBZ-vet, Karlsdorf-Neuthard; 2009 Promotion an der TiHo Hannover; ebenfalls 2009 Veröffentlichung des Buches „Osteopathie beim Hund“ mit Christiane Gräff; von 2007-2012 Dozentin am FBZ-vet; seit 2011 Dozentin im Deutschen Verband für Gebrauchshundsportvereine; 2015 Erlangung der Zusatzbezeichnung Physiotherapie und Rehabilitation bei Kleintieren. 